\title{
On the role of V1 in avoiding obstacles
}

\author{
Constanze Hesse ${ }^{1}$, Jutta Billino ${ }^{2} \&$ Thomas Schenk ${ }^{3 *}$
}

$02 / 11 / 2017$

Running head: Avoiding obstacles and misunderstandings

*Correspondence should be addressed to:

Dr. Thomas Schenk

Clinical Neuropsychology

Department Psychologie

Ludwig-Maximilians Universität München

Leopoldstr. 13

80802 München

Germany

Phone: +49 89-2180 3120

E-Mail: Thomas.schenk@psy.Imu.de

\footnotetext{
${ }^{1}$ School of Psychology, University of Aberdeen, Aberdeen, United Kingdom

${ }^{2}$ Experimental Psychology, Justus-Liebig-Universität, Giessen, Germany

${ }^{3}$ Clinical Neuropsychology, Ludwig-Maximilians-Universität München, Germany
} 
This commentary is the next in a chain of events. First, Striemer, Chapman, and Goodale (2009) argued, on the basis of a single case study testing a patient with hemianopia, that the primary visual cortex (V1) is not essential for successful obstacle avoidance behaviour. In the Cortex special issue on the "what and how-pathways", we (Ross, Schenk, Billino, MacLeod, \& Hesse, 2016) examined six patients suffering from hemianopia and came to the conclusion that obstacle avoidance during reaching crucially relies on input from V1. Subsequently, Striemer, Chapman, and Goodale (2017) have claimed that we misunderstood their original report. Clearly it is time that we get to the bottom of these misunderstandings and explore opportunities for finding common ground.

In the introduction of our study (Ross et al., 2016) we presented several reasons for why Striemer et al.'s (2009) single-case study requires corroboration from similar findings in a group of patients with damage to their occipital cortex but intact subcortical visual pathways. Striemer et al. (2017, section 2, $1^{\text {st }}$ paragraph) accept that our failure to replicate their original findings in a group of six patients (Ross et al., 2016) might constitute a serious challenge to the validity of their conclusions - but only if our study can be considered to be a fair test of their claims. In the rest of their commentary, Striemer et al. (2017) provide three main reasons for why our study does not provide such a fair test in their opinion. Firstly, they question the evidence from our patient study on the basis that our patients fail to fulfil the relevant conditions required for testing our hypothesis. Secondly, they suggest that we designed our experiments in ways that ensured a failure to replicate their original findings. Lastly, they argue that we misrepresented their position and attacked a straw man. In this commentary, we aim to demonstrate that (1) the disqualified evidence from our study can be recovered, (2) the methods used in our experiment have more redeeming aspects than granted; and finally, we assess the theoretical implications of Striemer et al.'s commentary for their original findings as well as for our study (3).

\section{The sample}

Striemer et al. (2009) demonstrated in a patient with hemianopia that the primary visual cortex (V1) is not essential for successful obstacle avoidance behaviour during reaching. We re-examined this claim and failed to find evidence for successful obstacle behaviour in any of our six patients suffering from hemianopia due to V1 damage. In their rebuttal, Striemer et al. (2017) start out by arguing that five patients of our sample were unsuitable for testing retained obstacle avoidance skills after V1 damage as they did not show signs of perceptual blindsight 
in the redundant target effect paradigm. Next, they suggest that the remaining patient (P4) was suffering from optic ataxia and take this as indication that P4's dorsal stream is partially damaged. On the basis of the assumption that optic ataxia invariably interferes with normal obstacle avoidance behaviour, Striemer et al. (2017) find it unsurprising that patient P4 also fails to show successful obstacle avoidance behaviour. In other words, obstacle avoidance requires two things: visual information about the position of the obstacles (which does not have to come from V1), and an intact dorsal stream. Without an intact dorsal system obstacleavoidance behaviour is not possible and the potential role of subcortical visual information for obstacle-avoidance cannot be tested.

In our view, Striemer et al. (2017)'s critique is based on two problematic assumptions. First, Striemer et al. (2017) assume that the ability to avoid obstacles requires perceptual blindsight as demonstrated by a redundant target effect task. However, there is no evidence for this position (cf. Corbetta, Marzi, Tassinari, \& Aglioti, 1990). Most importantly, action blindsight (in pointing and grasping tasks) has been found to exist in patients who do not show a redundant target effect (e.g., Whitwell, Striemer, Nicolle, \& Goodale, 2011). Their second argument is also problematic. Evidence from our patient P4 (the only patient who showed a redundant target effect) is dismissed on the grounds that her brain damage may have encroached upon dorsal stream territory. It is certainly true that P4's lesion extends beyond V1 and borders on areas that have been identified as part of the dorsal visuomotor system (e.g., Karnath \& Perenin, 2005). However, on the basis of the lesion data it is impossible to say whether or not the dorsal system has been functionally compromised as damage to a specific area can never predict the existence of a behavioural deficit with certainty (cf. Karnath, Berger, Küker, \& Rorden, 2004; Karnath \& Perenin, 2005). Striemer et al. (2017), therefore, put forward a second argument to support their claim that P4's dorsal stream is impaired. Specifically, they point out that in P4's blind field pointing errors are more pronounced for visual targets that are further away from her point of fixation. They then argue that such an eccentricity-dependent increase of pointing errors is a typical sign of optic ataxia. Thus, to Striemer et al. (2017) it seems clear that P4's dorsal stream must be damaged and therefore it is hardly surprising that P4 fails to respond to obstacles placed in her blind field. However, there are problems with this argument. Eccentricity-dependent increase of pointing errors can be interpreted as signs of optic ataxia and thus dorsal stream damage when those errors occur in a patient's intact field. However, the same is not necessarily true for targets presented in a patient's blind field. In fact, looking through the literature, it seems to be a quite common characteristic of blindsight pointing that errors increase with increasing target- 
eccentricity. In Figure 1, we present examples of blindsight pointing in three patients reported by other researchers. In these patients pointing errors also increased with target eccentricity even though their lesions were clearly focussed in the occipital cortex. Hence, eccentricitydependent increase of pointing errors in a patient's blind field is not necessarily indicative of dorsal-stream damage. Secondly, the assumption that dorsal stream damage and optic ataxia will inevitably destroy a patient's ability to avoid obstacles is refuted by studies on patient DF. In patient DF, significant thinning of cortical tissue in dorsal stream regions was documented (Bridge et al., 2013) and pronounced signs of optic ataxia were observed behaviourally (Hesse, Ball, \& Schenk, 2012, 2014). Nevertheless, patient DF was still found to avoid obstacles as efficiently as healthy individuals (Rice et al., 2006).

To summarise, the question is why Striemer et al's patient could avoid obstacles while our six patients could not. Their answer is that our patients were inappropriately selected as they either did not show perceptual blindsight or had a lesion that also compromised dorsal stream areas. In contrast, we would argue that the arguments put forward by Striemer and colleagues (2017) to dismiss our patients are not justified and based on problematic assumptions. As discussed in detail in our original report (Ross et al., 2016), we do however acknowledge the fact that most of our patients had more pronounced damage to occipital areas than Striemer et al.'s patient CB (who showed a Charles-Bonnet syndrome that has been associated with smaller occipital lesions). Hence, damage to occipital areas outside of V1 (e.g., visual association areas) may be responsible for the failure to find blindsight obstacle performance in our patients. However, by the same token, there is also the possibility that, in fact, spared V1 tissue in patient $\mathrm{CB}$ may have provided the input for the successful obstacle avoidance behaviour observed by Striemer and colleagues (2009). In other words, the findings of both studies leave some room for interpretation, and it seems fair to say that at the moment we have neither unequivocal evidence to support nor to refute the hypothesis of obstacle avoidance being independent of V1 input. It is worth noting that the issue of imperfect mapping of lesions onto cortical areas does not prevent us from drawing some conclusions from our findings, namely that a) spared subcortical processing is insufficient for blindsight obstacle-avoidance and that b) perception blindsight does not necessarily predict the occurrence of action blindsight. These implications show that we can gain important insights on the basis of our study. It thus seems premature to dismiss the evidence from our patients as irrelevant. Instead it is more fruitful to compare and contrast the evidence from our patients with that of other patients to gain a better understanding of blindsight phenomena. 


\section{The methods}

Striemer and colleagues (2017) ponder the possibility that aspects of our experimental design might have obscured successful obstacle-avoidance behaviour in our patients' blind field. First, they argue that we put the obstacles too close together and too close to the midline. As a result, patients might have collided with obstacles that were placed in their blind visual field. This problem was further compounded by using LEGO technology to fix the obstacles in place - converting what should be a harmless behavioural experiment into a health-and-safety hazard. Consequently, our patients were driven towards the adoption of a movement strategy that turned them insensitive to the location of any potential obstacles presented within their blind field. In addition, we were criticised for using obstacles that were too small, and thus hard to detect, and finally for choosing an outcome measure (lateral position of the hand at the depth where the obstacles were placed) that is unlikely to catch any effects of obstacleposition on hand position.

In our defence, we might argue that we anticipated the possibility of patients adopting a movement strategy which could make them insensitive to obstacles in their blind field. An obvious strategy would be to stay clear of the blind field by shifting the reaching path well into the intact visual field (see Ross et al., 2016 for a detailed discussion). To deal with this, we introduced a second obstacle-avoidance task. The second task employed two obstacles (one in the blind field and one in the seeing field) and should have discouraged patients from adopting reaching paths that ventured too far into the intact visual field. However, Striemer and colleagues (2017) argue that it is naïve to expect evidence of blindsight performance in a situation where signals coming from a patient's intact field are in competition with those from their blind field.

In the following we will argue that Striemer et al.'s critique is based on unfounded conjectures and ignores information reported in our article (Ross et al. 2016).

Let's start with their first critique. Obstacles in our study were moved too far toward the midline and thus too far into the workspace. This could increase the risk that patients collide with the obstacles and consequently adopt a strategy of moving away from the unseen part of their workspace. Such a strategy would render patients largely unresponsive to any obstacles present in their blind visual field. This possibility can be ruled out. Figures 5 and 6 (Ross et al., 2016) show the hand positions for each patient and every single trial at the time their hand passed the obstacle(s). Each and every one of those hand positions is at a safe distance from 
the obstacles. We can thus dismiss the argument that patients collided with the obstacles during our experiments.

It should also be noted that what Striemer and colleagues (2017) describe as a weakness of our study could in fact be seen as one of its strengths. Obstacles have to be close to a person's natural reaching path otherwise there is no need for that person to respond to its position. Obstacles that are placed far away from the reaching path will have little impact on the geometry of that path. Hence, by edging the obstacle closer to the reaching path we can enhance the potential impact of the obstacle's position and thereby enhance the sensitivity of our obstacle-avoidance test. It might also be noted that the true difference between positions used in our study and that by Striemer, Chapman, and Goodale (2009) is in fact much smaller than stated. Striemer et al. (2017) inflate this difference by using different reference points for the two respective studies (i.e. we reported the distance from the outer edges while Striemer and colleagues refer to the measures from the obstacle’s midpoint).

In addition, Striemer and colleagues (2017) argue that patients in our study were further induced to adopt an obstacle-insensitive reaching strategy by our use of LEGO cubes. They fear that our LEGO obstacles are more threatening than the obstacles used in their study. This fear is unfounded. The LEGO obstacles posed no risk to the participants. On collision, the obstacles would have easily toppled over without causing pain or harm to the participant. Incidentally, the NHS Grampian research ethics committee agreed with this assessment since they specifically asked us to employ obstacles that would easily fall over on light contact.

Yet, there are more concerns regarding our obstacle choice. In particular, Striemer and colleagues (2017) also pointed out that we used smaller obstacles than they did. This is true, but does not explain why we failed to find evidence of blindsight in our obstacle-avoidance experiments. The obstacles, while being smaller than those used by Striemer et al. (2009), were still substantial objects. The obstacles covered a space of $15 \mathrm{~cm}^{3}$ and were certainly in principle of sufficient size to evoke blindsight performance. In fact, we and others demonstrated blindsight performance with considerably smaller visual stimuli. Finally, our obstacle-avoidance measure was also criticised. We computed the lateral position of the hand at the moment it passed the obstacle(s). Striemer et al. (2017) state that this is not a sensitive measure. Instead they recommend using the hand's lateral position at the end of each reaching movement. Figure 7 (Ross et al., 2016) shows the reaching paths for one of our patients. This figure contains no indication that the hand position became more sensitive to the obstacle position(s) later during the reach. However, in the view of Striemer and colleagues, the only 
patient that merits further attention is P4. Therefore, we present her reaching paths for both obstacle configurations in this commentary (see Figure 2). P4 had a right hemianopia. In case of blindsight performance, we would expect that her hand shifts to the left when the right obstacle is shifted left and thus closer to the midline. Interestingly, for the one-obstacle condition the opposite is true. Her hand shifts towards the right, and thus closer to the obstacle, when the right obstacle is shifted inwards. Importantly, this is also true when we look at her hand-position at the end of her reach. A similar behaviour can be observed for the two-obstacle condition. In fact, in this condition, the paradoxical rightward shift is even more pronounced towards the end of her reaching movement.

Furthermore, it is questionable whether the end-position should be considered a valid, let alone better, measure of successful obstacle-avoidance behaviour than the hand-position at the time of passing the obstacles. We would argue that a capacity to take account of obstacle positions after the hand has already reached beyond those obstacles is about as useful as a navigation system for automatic parking which will provide the first warning signal after the car has crashed into that of a neighbour. We might therefore justifiably argue that obstacleinduced shifts that occur only in the final stage of the reaching trajectory (after the hand has passed the obstacle) are not evidence of successful obstacle-avoidance behaviour but actually indicate a dysfunctional obstacle-avoidance response.

In our opinion, the final criticism is the most surprising one. Striemer et al. (2017) argue that it is naïve to assume that patients with hemianopia might show action blindsight in a dualobstacle paradigm. In this paradigm patients have to take account of visual information from the blind and their intact field. According to Striemer and colleagues it is clear that even patients with blindsight capacity will disregard information from the blind field when at the same time visual information is also presented in their intact field. The basis for this assumption is not entirely clear. Possibly the idea is that if the sensorimotor system has the choice between information that is consciously available and information that is available on a subconscious level, it will always prefer conscious information and dismiss subconscious input. However, we know that this is not true from work on patients with extinction and patients with visual neglect. These patients were also examined under conditions where conscious and subconscious input was present at the same time. In fact, the paradigm used was identical to the one used in our study. McIntosh et al. (2004a,b) showed, that patients with unilateral neglect and a patient with visual extinction avoided objects on their neglected or extinguished side in a situation in which obstacles were also placed in their intact field. 
Furthermore, it should be noted that we introduced the dual-obstacle configuration in addition to the single-obstacle configuration, meaning that we provided patients with two opportunities to demonstrate their obstacle-avoidance capacities. This should have increased, and not diminished, our chances to find evidence of successful obstacle avoidance behaviour.

Let's sum up. Our methods are subtly different from those used by Striemer et al. (2009) but not worse. On the contrary, the changes were introduced to improve the chances to find evidence for blindsight performance. We used obstacle positions that were closer to the typical reaching path of our participants to increase the effect of changes in the obstacle's position. We also introduced a second obstacle-avoidance task with two obstacles to provide patients with an additional opportunity to demonstrate blindsight capacity in a paradigm whose sensitivity to detect the implicit use of visual information has previously been established (McIntosh et al., 2004a,b). The complete lack of any evidence for the successful obstacle avoidance in our patients' blind visual fields is therefore not a consequence of us using insensitive techniques but an indication that blindsight obstacle avoidance may be a rare capacity.

Thus, it seems we are again at an impasse. Striemer and colleagues (2017) suggest our findings can be dismissed. We argue that there is no good reason why our findings should not be taken seriously. Is there still a chance of finding some common ground? In fact, there may be more common ground than we originally assumed. In their comment on our study, Striemer and colleagues introduced a number of assumptions which cast a different light on their original finding and its interpretation. In our last section we will take a look at these assumptions and explore their implications in the wider context of the perception-action model.

\section{The interpretation}

Striemer et al. (2017) state that in our study (Ross et al., 2016) we build up and attacked a straw man that bears little resemblance to their original hypothesis. In their view, our 
interpretation of their hypothesis is overloaded with assumptions that are unrealistic ${ }^{1}$. In this section we identify and remove step by step each of those controversial assumptions. We will characterize the interpretation that emerges and leave it to the reader to evaluate the relevance of the resulting interpretation.

In their original report, Striemer et al. (2009) refer to the notion of two separate visual streams mediating vision for action and vision for perception (Goodale \& Milner, 1992). The task of avoiding obstacles is assigned to the dorsal visual stream but what remained unclear was the source of visual input to the dorsal stream. More specifically, they asked whether input from V1 is required for successful obstacle avoidance (Striemer et al., 2009, p. 15996). Having found that their patient CB is sensitive to the position of obstacles presented in his blind field they conclude that "visuomotor networks mediating reaching do not require V1 input to integrate the location of potential obstacles when planning these complex movements" (Striemer et al., 2009, p. 16000). In our interpretation, we took this to mean that normal obstacle avoidance behaviour can be carried out without input from V1. Such a statement, if backed up by compelling empirical evidence, is of significant theoretical importance. It seems to indicate that at least some complex forms of visuomotor behaviour are truly independent of the classical geniculate-V1 input stream. This feature would set visuomotor processes apart from perceptual processes and thereby provide further support for the overarching hypothesis that processing streams for vision for action and vision for perception are distinct and separate. However, the key difference is "true independence”. One way to examine whether a given visual behaviour is truly independent of V1 is to test whether that visual behaviour remains unaltered when V1-input is removed. More specifically, if it could be shown that for example visual position discrimination under blindsight conditions (performance in blind field of a patient with V1 lesion) is as good as under normal conditions (intact field of the patient or performance in healthy controls) we would have a good reason to argue that position discrimination does not need V1 input and is thus independent of V1-input. As a matter of fact this is not true for most blindsight studies. Regardless of whether we look at perceptual

\footnotetext{
${ }^{1}$ Striemer et al. (2017) were particularly incensed by our statement that the effects observed in their patient CB might constitute a chance finding. We would like to remind readers that several high-impact publications in recent years alerted us to the surprising prevalence of published chance-findings (Open-Science-Collaboration, 2015). In fact, we all typically accept that $5 \%$ of our significant findings will be produced by chance. Chance can therefore never be ruled out as a potential explanation. All we wanted to say is this: We tried our best to identify factors that might explain why our findings conflict with those of Stiemer et al. (2009). However, it is also possible that such factors do not exist because the effects may have been caused by chance. This was not just idle speculation. We had found in our own data that one of our patients showed significant but paradoxical shifts towards the obstacles. This alerted us to the possibility that chance fluctuations in a patient's behaviour might give rise to significant but spurious effects.
} 
(e.g., Ptito, Lepore, Ptito, \& Lassonde, 1991; Weiskrantz, Warrington, Sanders, \& Marshall, 1974) or visuomotor paradigms (e.g., Pöppel, Held, \& Frost, 1973; Whitwell et al., 2011), blindsight performance is usually worse than the patient's performance in the intact visual field. However, what was unclear until now is whether or not obstacle-avoidance provides an exception to this rule. If yes, this would provide the first example of a complex visuomotor behaviour that is truly independent of V1 input. In fact, we thought this was the novel claim put forward by Striemer and colleagues (2009). Accordingly, we expected that it should not be too difficult to find evidence of blindsight obstacle avoidance in other patients with V1 lesions. We were, therefore, surprised to find that not a single patient in our sample responded to obstacles placed in their blind visual field. Now we learned that our interpretation of the original study of Striemer et al. (2009) was wrong. Patient CB responded to the position of obstacles placed in his blind field, but his response was abnormal ${ }^{2}$. The obstacle-induced shift observed in his reaching trajectories came significantly later than in healthy controls. Consequently, we have to conclude that there is no evidence that obstacle-avoidance can proceed normally in the absence of V1 input.

Nevertheless, the findings from Striemer et al., (2009) could be taken as evidence that obstacle avoidance is potentially less reliant on V1 input than other behavioural tasks. CB's preserved ability to respond to obstacles in his blind field contrasted with his inability to point to targets in his blind field. Thus, it might be hypothesised that obstacle avoidance is less reliant on V1 input than pointing. In fact, this hypothesis provided the original motivation for our study (Ross et al., 2016). However, our findings refute this hypothesis. We describe one patient (P4) who showed blindsight in a pointing task but not in an obstacle-avoidance paradigm. One might also argue that the real relevance of the original findings and our study lies in the observation that blindsight performance can dissociate for different tasks, indicating that blindsight in different tasks is served by separate neuroanatomical loops within the primate visual system. Within the framework of the perception-action model (Goodale \& Milner, 1992) the most interesting dissociation is the one between perceptual and visuomotor tasks. Do we have evidence of separate blindsight loops for perceptual and action blindsight? Currently evidence on this is lacking (see also Danckert \& Rossetti, 2005 for discussion). A systematic study comparing blindsight performance for a large variety of perceptual and visuomotor tasks conducted in the same group of patients has to our knowledge not yet been carried out. However, it is clear that Striemer and colleagues (2017) do not expect such

\footnotetext{
${ }^{2}$ Striemer et al. (2009) noted this abnormality but discounted it as a consequence of impaired depth perception.
} 
dissociations. In fact, they pointed out that it would be naïve to expect action blindsight in patients who do not also show blindsight in other tasks such as the redundant target paradigm. Why is this expectation naïve? Presumably, because the pathway underlying the redundant target paradigm (attentional blindsight) is a vital part of the pathway underlying action blindsight. If the first pathway is impaired, the second will also be compromised. We are thus forced to conclude that in Striemer et al.'s view blindsight loops for action blindsight are not necessarily distinct from those involved in other forms of blindsight.

One other interpretation of the findings presented by Striemer et al., (2009) was provided by Goodale (2011). He suggested visual signals bypassing V1 provide an important source of input to the dorsal stream. He emphasised that these extra-V1 pathways are not just a 'backup' option for when V1 is lesioned "but play a central role in the control of actions such as reaching and grasping.” (Goodale, 2011, p. 1577). This interesting hypothesis is hard to reconcile with the assumption of Striemer et al., (2017) that when extra-V1 signals compete with V1 signals, the extra-V1 signals have insufficient weight to have a discernible impact on observable behaviour. We would argue that this assumption is incommensurate with Goodale (2011)'s hypothesis that extra-V1 signals play a central role in the normal guidance of reaching behaviour given that in the normal case V1 and extra-V1 signals are always concurrently available.

We can now ask ourselves what is the remaining relevance of the findings presented by Striemer et al., (2009) once we stripped it of all those unwarranted overinterpretations? According to Striemer et al. (2017), CB's ability to shift his reaching trajectory in response to obstacles placed in his blind field should not be taken as evidence that the capacity for obstacle-avoidance is independent of V1 input. Nor should it be taken as evidence that action blindsight and non-action blindsight are served by distinct subcortical loops. Finally, CB's behaviour does also not provide support for the claim that extra-V1 signals play an important role in the normal control of reaching behaviour. Thus, taking the clarifications provided by Striemer et al. (2017) into account, the most we may possibly say is that in the absence of competing signals from V1, extra-V1 signals can drive some form of diminished but still significant obstacle-avoidance response. In short, the same kind of compromised but significant implicit visual sensitivity that has been demonstrated in patients' blind fields for pointing, position, orientation and colour discrimination, word recognition and numerous other tasks (Cowey, 2010) may also be observed for obstacle-avoidance performance. This conclusion is not particularly contentious. 


\section{Acknowledgements}

Thomas Schenk was supported by grants from the German Research Foundation (Deutsche Forschungsgemeinschaft, DFG, grant no's: DFG-SCHE 735/2-1 and DFG-SCHE 735/3-1). Jutta Billino was supported by a grants from the German Research Foundation (Deutsch Forschungsgemeinschaft, Collaborative Research Centre SFB/TRR 135: Cardinal Mechanisms of Perception). We wish to thank Edward De Haan and two anonymous reviewers whose thoughtful comments helped to improve the manuscript. 


\section{References}

Bridge, H., Thomas, O. M., Minini, L., Cavina-Pratesi, C., Milner, A. D., \& Parker, A. J. (2013). Structural and functional changes across the visual cortex of a patient with visual form agnosia. Journal of Neuroscience, 33(31), 12779-12791. doi: 10.1523/jneurosci.4853-12.2013

Corbetta, M., Marzi, C., Tassinari, G., \& Aglioti, S. (1990). Effectiveness of different task paradigms in revealing blindsight. Brain, 113(3), 603-616.

Cowey, A. (2010). The blindsight saga. Experimental Brain Research, 200(1), 3-24. doi: 10.1007/s00221-009-1914-2

Danckert, J., Revol, P., Pisella, L., Krolak-Salmon, P., Vighetto, A., Goodale, M. A., \& Rossetti, Y. (2003). Measuring unconscious actions in action-blindsight: exploring the kinematics of pointing movements to targets in the blind field of two patients with cortical hemianopia. Neuropsychologia, 41(8), 1068-1081.

Danckert, J., \& Rossetti, Y. (2005). Blindsight in action: what can the different sub-types of blindsight tell us about the control of visually guided actions? [Review]. Neuroscience and Biobehavioral Reviews, 29(7), 1035-1046. doi: 10.1016/j.neubiorev.2005.02.001

Goodale, M. A. (2011). Transforming vision into action. Vision Research, 51(13), 1567-1587.

Goodale, M. A., \& Milner, A. D. (1992). Separate visual pathways for perception and action. Trends in Neurosciences, 15(1), 20-25.

Hesse, C., Ball, K., \& Schenk, T. (2012). Visuomotor performance based on peripheral vision is impaired in the visual form agnostic patient DF. Neuropsychologia, 50(1), 90-97. doi: 10.1016/j.neuropsychologia.2011.11.002

Hesse, C., Ball, K., \& Schenk, T. (2014). Pointing in visual periphery: Is DF's dorsal stream intact? PloS One, 9(3), e91420. doi: 10.1371/journal.pone.0091420

Karnath, H.-O., Berger, M. F., Küker, W., \& Rorden, C. (2004). The anatomy of spatial neglect based on voxelwise statistical analysis: a study of 140 patients. Cerebral cortex, 14(10), 1164-1172.

Karnath, H.-O., \& Perenin, M.-T. (2005). Cortical control of visually guided reaching: evidence from patients with optic ataxia. Cerebral Cortex, 15(10), 1561-1569.

McIntosh, R. D., McClements, K. I., Dijkerman, H. C., Birchall, D., \& Milner, A. D. (2004a). Preserved obstacle avoidance during reaching in patients with left visual neglect. [Article]. Neuropsychologia, 42(8), 1107-1117. doi: 10.1016/j.neuropsychologia.2003.11.023

McIntosh, R. D., McClements, K. I., Schindler, I., Cassidy, T. P., Birchall, D., \& Milner, A. D. (2004b). Avoidance of obstacles in the absence of visual awareness. [Article]. Proceedings of the Royal Society of London Series B-Biological Sciences, 271(1534), 15-20. doi: 10.1098/rspb.2003.2545

Open-Science-Collaboration. (2015). Estimating the reproducibility of psychological science. Science, 349(6251), aac4716.

Pöppel, E., Held, R., \& Frost, D. (1973). Residual visual function after brain wounds involving the central visual pathways in man. Nature, 243(5405), 295-296.

Ptito, A., Lepore, F., Ptito, M., \& Lassonde, M. (1991). Target detection and movement discrimination in the blind field of hemispherectomized patients. Brain, 114(1), 497-512.

Rice, N. J., McIntosh, R. D., Schindler, I., Mon-Williams, M., Démonet, J. F., \& Milner, D. A. (2006). Intact automatic avoidance of obstacles in patients with visual form agnosia. Experimental Brain Research, 174, 176-188.

Ross, A. I., Schenk, T., Billino, J., MacLeod, M. J., \& Hesse, C. (2016). Avoiding unseen obstacles: Subcortical vision is not sufficient to maintain normal obstacle avoidance behaviour during reaching. . Cortex. doi: 10.2016/j.cortex.2016.09.010.

Striemer, C. L., Chapman, C. S., \& Goodale, M. A. (2009). Real-time obstacle avoidance in the absence of primary visual cortex. Proceedings of the National Academy of Sciences of the United States of America, 106(37), 15996-16001. 
Striemer, C. L., Chapman, C. S., \& Goodale, M. A. (2017). The role of non-conscious visual processing in obstacle avoidance: A commentary on Ross et al., 2016. Cortex. doi: 10.1016/j.cortex.2017.03.024

Weiskrantz, L., Warrington, E. K., Sanders, M., \& Marshall, J. (1974). Visual capacity in the hemianopic field following a restricted occipital ablation. Brain, 97(1), 709-728.

Whitwell, R. L., Striemer, C. L., Nicolle, D. A., \& Goodale, M. A. (2011). Grasping the non-conscious: Preserved grip scaling to unseen objects for immediate but not delayed grasping following a unilateral lesion to primary visual cortex. Vision research, 51(8), 908-924. 


\section{Figure Legends}

Figure 1: Single Patient data showing the pointing errors of three different patients in a localisation tasks as reported by two different studies. A) Patient SJ as reported by Whitwell et al. (2011) in Figure 2. Note that we used the regression line to estimate the values as the mean values were unavailable in this study. Data was digitized using the freeware PlotDigitizer (Free Software Foundation, Inc.) and converted in cm (viewing distance of $40 \mathrm{~cm}$ ). B) Pointing errors of Patient JR and C) of Patient YP as reported by Danckert et al. (2003) in middle panel of Figure 3 (intact visual fields) and Figure 4 (blind visual fields). Note that we converted the target positions provided in degrees of visual angle (at a viewing distance of $30 \mathrm{~cm}$ ) to calculate the pointing errors in $\mathrm{cm}$. Data was digitized using the freeware PlotDigitizer (Free Software Foundation, Inc.).

Figure 2: Time-normalised hand trajectory plots (averaged across trials) as recorded for the one-obstacle condition (left panel) and the two-obstacle condition (right panel) for P4 who suffered from a right hemianopia as reported in Ross et al. (2016). Obstacles were placed at $200 \mathrm{~mm}$ in y-direction. For details see text. 
A)

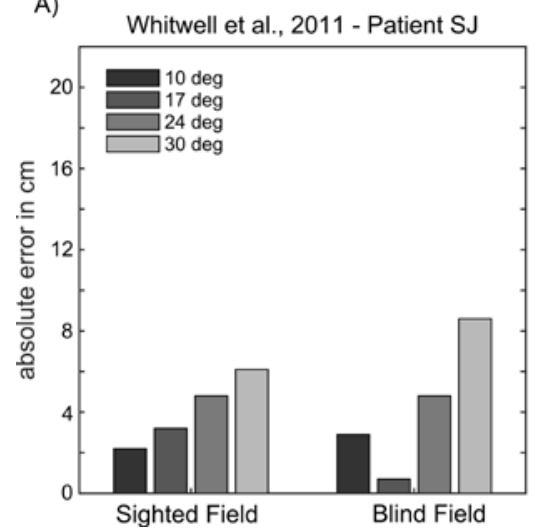

B)

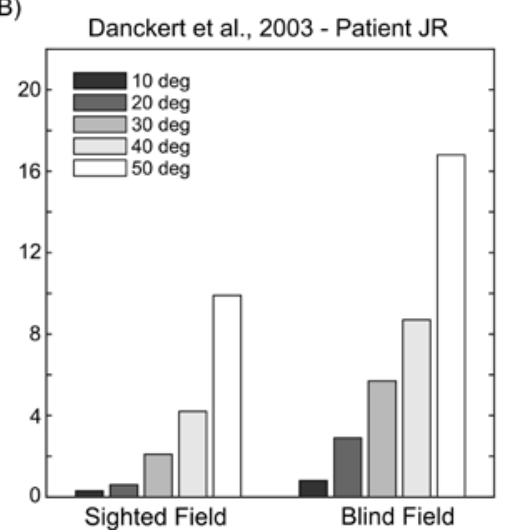

C)

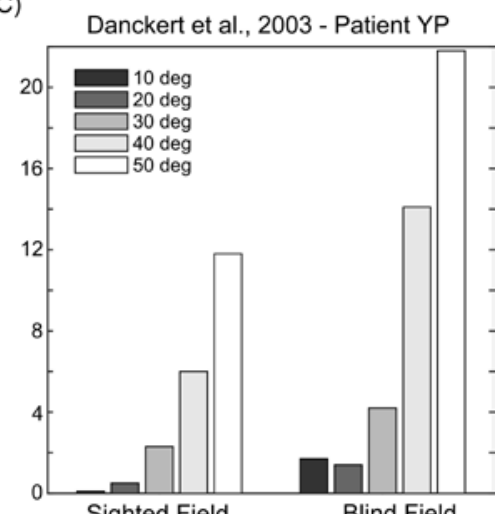

Figure 1 
One-obstacle condition

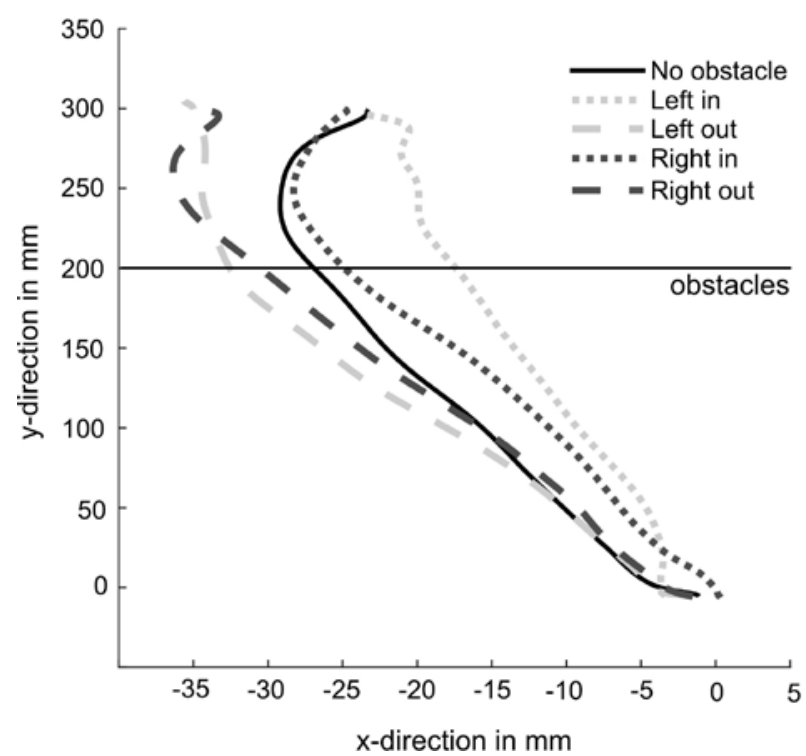

Two-obstacle condition

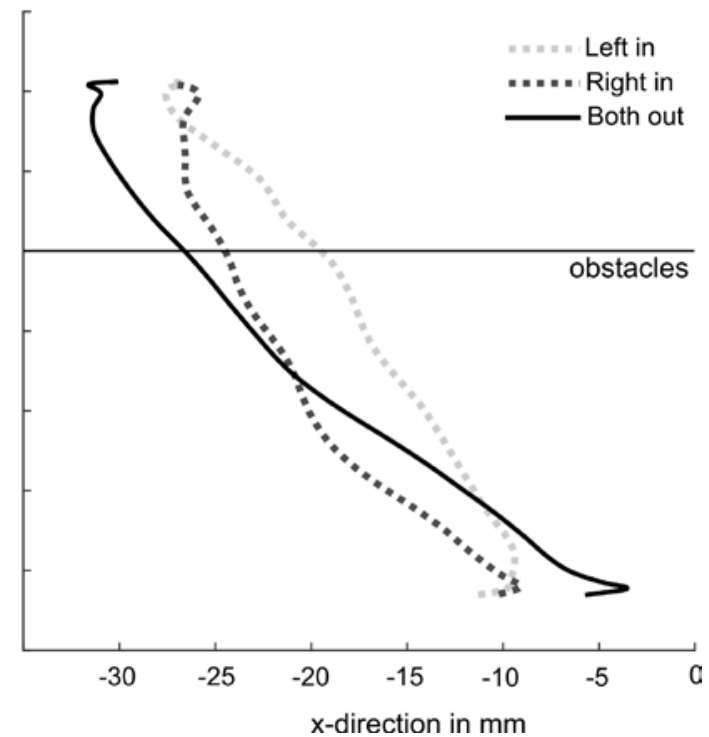

Figure 2 